\title{
Nefrotoxicidad por contrastes yodados en estudios de tomografía computarizada a pacientes ambulatorios diabéticos en tratamiento con metformina
}

\section{Nephrotoxicity due to iodine contrasts in computerized tomography studies of diabetic outpatients on metformin}

\author{
H. Gómez Herrero', C. De Arriba Villamor ${ }^{1}$, M. Buldain Parra ${ }^{1}$, M. Arraiza Sarasa ${ }^{1}$
}

\section{RESUMEN}

Fundamento. Determinar la incidencia de nefropatía inducida por contraste (NIC) en un grupo de pacientes diabéticos en tratamiento con metformina a los que se realiza un estudio de tomografía computarizada (TC) con contraste yodado intravenoso (civ)

Material y Métodos. Estudio prospectivo de pacientes diabéticos incluidos en el protocolo para la prevención de la acidosis láctica a los que se hace seguimiento clínico y analítico tras la realización de una TC con civ.

Resultados. Se recogieron 98 casos. En los pacientes sin insuficiencia renal previa la incidencia de NIC es del $0 \%$. En los pacientes con insuficiencia renal previa la incidencia de NIC es del $4,7 \%$.

Conclusiones. El riesgo de NIC en pacientes diabéticos sin insuficiencia renal a los que se administra contraste yodado es mínimo. Las recomendaciones de retirar la metformina para evitar la acidosis láctica en pacientes a los que se administra civ se podría limitar a los pacientes con alteración de la función renal.

Palabras clave. Nefropatía inducida por contraste. Tomografía computarizada. Contraste yodado. Metformina.

\begin{abstract}
Background. To determine the incidence of contrastinduced nephropathy (CIN) among diabetic patients treated with Metformin who underwent computerized tomography (CT) scan with iodinated contrast media.

Methods. Prospective study of diabetic patients enrolled in a lactic acidosis prevention protocol in whom clinical and analytical follow up after CT scan with intravenous contrast was performed.

Results. In this study, 98 cases were collected. The incidence of $\mathrm{CIN}$ in diabetic patients without prior renal failure was $0 \%$. In patients with previous renal failure the incidence of CIN was $4.7 \%$.

Conclusions. The risk of CIN in diabetic patients with no renal failure undergoing intravenous contrast administration is minimal. Recommendations to stop Metformin to avoid lactic acidosis in patients undergoing intravenous contrast administration may be restricted to patients with abnormal renal function.
\end{abstract}

Key words. Contrast-induced nephropathy. Computed tomography. Iodinated contrast. Metformin.
1. Servicio de Radiología. Complejo Hospitalario de Navarra. Pamplona.

Recepción: 2 de noviembre de 2012

Aceptación provisional: 17 de enero de 2013

Aceptación definitiva: 8 de febrero de 2013

\section{Correspondencia:}

Helena Gómez Herrero

Servicio de Radiología

Complejo Hospitalario de Navarra

$\mathrm{C} /$ Irunlarrea, 3

31008 Pamplona

E-mail: hgomezhe@navarra.es 


\section{INTRODUCCIÓN}

La primera referencia de la nefropatía inducida por contraste yodado (NIC) es de $1954^{1}$, y se define como "la condición en la cual hay un empeoramiento de función renal en los días inmediatamente posteriores a la administración intravascular de contraste en ausencia de otra causa de disfunción renal". Su incidencia en la bibliografía oscila entre el 0,6 y el $50 \%^{2}$. Se considera a la nefropatía, sobre todo asociada a diabetes, el más importante factor de riesgo para el desarrollo de $\mathrm{NIC}^{3,4}$. Otros factores de riesgo que se postula que tienen un papel en el desarrollo de NIC son la deshidratación, la insuficiencia cardíaca, la edad avanzada, el uso concomitante de otras drogas nefrotóxicas, la inestabilidad hemodinámica, la hipotensión y la anemia ${ }^{5}$. La NIC es una alteración reversible en la mayoría de los casos, desconociéndose la repercusión exacta sobre la morbimortalidad.

El criterio más aceptado para valorar si existe NIC es un aumento de la creatinina sérica $>0,5 \mathrm{mg} / \mathrm{dl}$ (según criterios absolutos) o $>25 \%$ (según criterios relativos) respecto a las condiciones basales. Están por determinar otros criterios como la disminución de la tasa de filtración glomerular (TFG) ${ }^{5}$.

La mayoría de los datos de NIC que se disponen son de estudios de cardiología intervencionista y hay pocos estudios que valoren la NIC en estudios de tomografía computarizada (TC).

Con el gran desarrollo de las pruebas de imagen médicas se han extendido de forma considerable el uso de pruebas como la TC, muchas de ellas realizadas con contraste yodado intravenoso (civ). También han evolucionado considerablemente los contrastes yodados, hasta los empleados actualmente, no iónicos o hipoosmolares, con la disminución de sus reacciones adversas, nefrotoxicidad incluida.

Por todos estos motivos la NIC ha sido últimamente motivo de revisión en la bibliografía médica.

Como efecto adverso secundario a la nefrotoxicidad del contraste yodado, en pacientes diabéticos en tratamiento con metformina, está descrita la posibilidad de acidosis láctica, por la acumulación de este fármaco al reducirse su eliminación renal en el caso de que se produzca NIC.

La acidosis láctica asociada a la metformina es una condición metabólica poco frec ( 8 casos/100.000 pac/año) pero grave, con mortalidad de $20-50 \%^{6}$, de ahí la importancia de establecer recomendaciones para su prevención. Existe una gran variabilidad en las recomendaciones respecto a la retirada de la metformina previa a la administración de contraste ${ }^{7}$.

La mayoría de las pruebas que requieren civ actualmente son estudios de TC en pacientes ambulatorios, muchos de ellos diabéticos en tratamiento con metformina, por la alta incidencia de esta enfermedad y este fármaco en la población general.

Se contrastan los resultados con las recomendaciones de la última edición de la Guía para el Manejo de los Contrastes de la European Society of Urogenital Radiology $\left(\right.$ ESUR) ${ }^{8}$.

La necesidad de valorar la incidencia de NIC en estos pacientes así como identificar a la población de riesgo sobre la que realizar medidas de control y preventivas ha sido la motivación inicial para la realización de este estudio.

\section{MATERIAL Y MÉTODOS}

Estudio prospectivo de pacientes consecutivos a los que se realiza un estudio de TC con civ en un centro médico para pacientes ambulatorios en el período de enero a agosto del 2012.

Se recogieron datos de 102 estudios de TC con contraste. Cuatro se desestimaron porque los pacientes no se realizaron la analítica posterior, quedando un total de 98 casos.

Los pacientes eran diabéticos en tratamiento con metformina, incluidos en el protocolo de metformina y contraste realizado en nuestro centro para evitar la acidosis láctica. A los pacientes se les daba información verbal y escrita del protocolo que consistía en suspender este medicamento tras la administración de civ y decidir su reintroducción tras la realización de una analítica de función renal. Si la función renal del paciente estaba alterada previo a la 
realización de la TC, la metformina se suspendía 2 días antes de la prueba.

Previo a la realización de la TC se recogieron datos de función renal de los pacientes la cifra de creatinina y la tasa de filtración glomerular (TFG), que se valoró según la fórmula MDRD-4IDMS.

Tras la realización de la TC se les indicaba a los pacientes que se realizaran una analítica de control de función renal (creatinina y TFG) aproximadamente 2 días tras la prueba.

Se recogieron los datos demográficos de los pacientes y la cantidad de contraste empleado. El tipo de contraste utilizado fue el ioversol $320 \mathrm{mg} / \mathrm{mL}$ (Optiray ${ }^{\circledR}$ ).

El seguimiento se hizo con la historia clínica informatizada valorando los datos de la analítica postprueba, complicaciones médicas en el plazo de un mes tras la prueba que motivaran consultas médicas, hospitalización o visitas a urgencias en relación con alteración de la función renal o alteración de la glucemia.

El criterio de alteración de función renal previa fue de creatinina mayor de 1,2 $\mathrm{mg} / \mathrm{dL}$ y/o TFG $<60 \mathrm{ml} / \mathrm{min} / 1,72 \mathrm{~m}$. El criterio de NIC empleado fue el de la elevación de la cifra de $\mathrm{cr}>0,5 \mathrm{mg} / \mathrm{dL}$.

Ninguno de los pacientes recibió medicación de profilaxis de nefrotoxicidad. La pauta de hidratación consiste en hidratación oral. Al paciente se le insistió sobre la ingesta de líquidos en las 24 horas posteriores.

\section{RESULTADOS}

Se estudiaron 98 casos, con una media de edad de 67,7 (DS 12) y una relación hombre/mujer de 57/41. La media de la cantidad de contraste que recibieron fue de $100 \mathrm{~mL}$, no superando en ningún caso los $150 \mathrm{~mL}$. La fecha de la analítica preprueba fue muy variable, con una mediana de 52,5 días. La fecha de la analítica postprueba se realizó con una mediana de 2,5 días.

De los 98 casos, 77 tenían la función renal normal previa a la prueba y 21 alterada. De esos 21 pacientes, 15 tenían alterada la creatinina y la TFG y 6 sólo la TFG. En los casos de función renal alterada, la media de los valores de creatinina fue de $1,5 \mathrm{mg} /$ dL y de TFG de 47. La alteración de la función renal de los pacientes no fue en ningún caso de $\mathrm{cr}>2$ o TFG $<30$.

Se dividió a los pacientes en dos grupos: grupo 1, función renal normal, y grupo 2 , función renal alterada. Los datos de la analítica postprueba de los dos grupos se exponen en la tabla 1 donde se reflejan los pacientes que no empeoraron la función renal, los pacientes que empeoraron la función renal sin cumplir los criterios de NIC (elevación de los niveles de $\mathrm{cr}<0,5$ $\mathrm{mg} / \mathrm{dL}$ ) y los que cumplieron criterios de NIC.

En el grupo 1, pacientes con función renal normal ( $\mathrm{n}=77)$, el $96,1 \%$ de los pacientes no empeoraron la función renal, un $3,8 \%$ sufrieron una leve alteración y no hubo ningún caso de NIC.

En el grupo 2, pacientes con función renal alterada ( $\mathrm{n}=21)$, el $47,6 \%$ no empeoraron la función renal, un $47,6 \%$ sufrieron una leve alteración de función renal y hubo un caso de NIC.

Tabla 1. Datos de función renal tras la administración de contraste.

\begin{tabular}{lccc}
\hline & $\begin{array}{c}\text { Grupo 1 n: 77 } \\
\text { creatinina<1,2mg/dL } \\
\text { TFG>60 ml/min/1,72 m }\end{array}$ & $\begin{array}{c}\text { Grupo 2 n: 21 } \\
\text { creatinina }>\mathbf{1 , 2 m g} / \mathbf{d L} \\
\text { y/o TFG }<\mathbf{6 0} \mathbf{~ m l} / \mathbf{m i n} / \mathbf{1 , 7 2} \mathbf{~ m}\end{array}$ & $\begin{array}{c}\text { Total } \\
\mathbf{n : ~ 9 8}\end{array}$ \\
\hline No empeoramiento de función renal & $74 / 77(96,1 \%)$ & $10 / 21(47,6 \%)$ & $84 / 98(85,7 \%)$ \\
\hline $\begin{array}{l}\text { Leve alteración de función renal } \\
\text { Aumento de creatinina<0,5 mg/dL }\end{array}$ & $3 / 77(3,8 \%)$ & $10 / 21(47,6 \%)$ & $13 / 98(13,2 \%)$ \\
\hline $\begin{array}{l}\text { NIC } \\
\text { Aumento de creatinina }>0,5 \mathrm{mg} / \mathrm{dL}\end{array}$ & $0 / 77(0 \%)$ & $1 / 21(4,7 \%)$ & $1 / 98(1 \%)$ \\
\hline
\end{tabular}

NIC: nefropatía inducida por contraste. TFG: tasa de filtración glomerular 
Dada la distribución no normal de la muestra (Test de Kolmogorov-Smirnov $<0.001$ ) para realizar la comparación entre valores de creatinina utilizamos la prueba de Wilcoxon de los rangos con signo de muestras relacionadas, la cual no llegó a encontrar diferencias significativas entre los valores de creatinina basal y tras el contraste endovenoso. Cuando seleccionamos a los pacientes con deterioro de la función renal, solo en aquellos con creatinina basal $>1,7$ el test se acercaba a las diferencias significativas $(\mathrm{p}=0,08)$.

En el seguimiento de los pacientes ninguno requirió ningún tipo de atención médica, en relación a la alteración de la función renal. Dos pacientes sufrieron alteración de la glucemia que requirió atención en urgencias y hospitalización, respectivamente.

\section{DISCUSIÓN}

Algunos artículos ${ }^{2,5}$ cuestionan la sobrestimación de la NIC, motivada de la extrapolación de estudios cardíacos, en enfermos hospitalizados, con más factores de riesgo y mayor dosis de contraste. El riesgo de NIC en TC respecto a las pruebas de intervencionismo cardíaco se considera menor por la administración intravenosa y no arterial, la menor cantidad de contraste usado y la ausencia de los embolismos de colesterol, complicación descrita en estudios coronarios ${ }^{5}$.

En una revisión de 54 estudios $^{9}$ para valorar NIC, solo 8 eran de TC de los que solo un estudio incluyó pacientes con función renal normal y solo un estudio incluyó un paciente al que se realizó pruebas de TC programadas. En otra revisión ${ }^{10}$ para la valoración de NIC en pacientes con insuficiencia renal a los que se les realizaba una TC con contraste se obtuvo una incidencia global del 5\%, muy próxima al valor de 4,7\% en nuestra breve serie.

Un estudio ${ }^{11}$ comparó valores de creatinina de pacientes a los que se había realizado una TC sin y con contraste, no demostrándose variaciones significativas, lo que apoyaría la existencia de fluctuaciones fisiológicas de la creatinina, por lo que éste no sería un indicador para valoración de alteración de función renal en variaciones le- ves. También hay que tener en cuenta que la alteración analítica de aumento de creatinina no siempre tiene repercusión clínica.

El protocolo actual en nuestro servicio para la prevención de la acidosis láctica se ajusta a las recomendaciones de la primera guía del Comité para la Seguridad de los Medios de contraste de ESUR de 1999.

En nuestra serie ninguno de los pacientes con $\mathrm{TFG}>60$ vio deteriorada de forma significativa su función renal y el único caso de NIC fue en un paciente con una TFG previa de $44 \mathrm{ml} / \mathrm{min}$. Pese a no llegar a encontrar diferencias significativas a nivel estadístico (lo cual se debe probablemente al escaso tamaño muestral), la observación global (Tabla 1) de los resultados parece apuntar a que solo en aquellos pacientes con una creatinina $>1,2 \mathrm{mg} / \mathrm{dL}$ parece tener impacto a nivel clínico el uso de civ. Esto concordaría con las últimas recomendaciones de las principales guías radiológicas para pacientes diabéticos con función renal normal donde se relajan las medidas de prevención en relación con la metformina ${ }^{7}$. También cabe reseñar que mientras sólo hubo un caso de NIC sin repercusión clínica, hubo 2 pacientes que requirieron atención médica por modificación de la pauta de tratamiento antidiabético.

La última revisión ${ }^{5}$ de 2011 de la guía ESUR para medios de contraste es aún más permisiva ya que se concluye que sólo los pacientes con TFG $<45 \mathrm{ml} / \mathrm{min}$ tienen riesgo significativo de sufrir NIC, al administrar contraste intravenoso, por lo que es en este grupo donde se recomienda suspender la metformina (Tabla 2).

Tras las últimas revisiones bibliográficas se ha visto que el riesgo de NIC es mucho menor en estudios de TC que en revisiones previas sobre estudios coronarios. Este riesgo disminuye en pacientes con nula o leve alteración de la función renal a los que se va a realizar un estudio programado de forma ambulatoria ${ }^{12}$.

Teniendo en cuenta la creciente utilización de pruebas diagnósticas basadas en los contrastes yodados es importante que, tanto los clínicos solicitantes de la prueba, como los radiólogos que las realizan, conozcan los posibles efectos secundarios de los contrastes y sus interacciones para poder tomar las medidas preventivas oportunas. 
Tabla 2. Recomendaciones de la última edición de la guía de la European Society of Urogenital Radiology (ESUR) para la administración de contrastes en pacientes en tratamiento con metformina

\begin{tabular}{lcc}
\hline & Vía intravenosa & Vía intraarterial \\
\hline No stop metformina & TFG $>45$ & TFG $>60$ \\
\hline $\begin{array}{l}\text { Stop metformina 48 h antes del contraste y reintroducir sólo si la } \\
\text { función renal no se ha deteriorado }\end{array}$ & TFG<45 & TFG 30-59 \\
\hline
\end{tabular}

TFG: tasa de filtración glomerular en $\mathrm{ml} / \mathrm{min} / 1,72 \mathrm{~m}$.

Futuros estudios para valoración de NIC deberían incluir series más grandes de pacientes con insuficiencia renal y teniendo en cuenta datos clínicos en el seguimiento como hospitalización por fallo renal, necesidad de diálisis y no sólo las alteraciones analíticas.

\section{BIBLIOGRAFÍA}

1. Bartels ED, Baun GC, Gammeltoft A, Gjorup PA. Acute anuria following intravenous pyelography in a patient with myelomatosis. Acta Med Scand 1954; 150: 297-302.

2. Arana E, Catalá-López F. Nefropatía inducida por contraste en pacientes de riesgo con insuficiencia renal explorados con tomografía computarizada: revisión sistemática y metaanálisis de ensayos clínicos aleatorizados. Med Clin (Barc) 2010; 135: 343-350.

3. Parfrey PS, Griffiths SM, Barrett BJ, Paul M, Genge M, Withers J et al. Contrast materialinduced renal failure in patients with diabetes mellitus, renal insufficiency, or both. A prospective controlled study. New Engl J Med 1989; 320: 143-149.

4. Mc Cullogh PA, Stacul F, Becker CR. ContrastInduced Nephropathy (CIN) Consensus Working Panel: executive summary. Rev Cardiovasc Med 2006; 7: 177-197.

5. Stacul F, Van der Molen A, Reimer P, Webb J, Thomsen H, Morcos S et al. Contrast induced nephropahy: update ESUR Contrast Media Safety Committee guidelines. Eur Radiol 2011; 21: 2527-2541.
6. Macías-Robles M.D, Macías-Bobes C, Yano-EscuDERo R, Fernández-Diéguez O, Álvarez-Lecue O. Acidosis láctica por metformina desencadenada por una insuficiencia renal aguda. An Sist Sanit Navar 2011; 34: 115-118.

7. Goergen S, Rumbold G, Compton G, Harris C. Systematic Review of current guidelines, and their evidence base, on risk of lactic acidosis after administration of contrast medium for patients receiving metformin. Radiology 2010; 254: 261-269.

8. Renal adverse reactions to iodinated contrast media (2008) Contrast mdia safety guidelines of the Contrast Media Safety Committee of the European Society of Urogenital Radiology, version 8.0. http://www.esur.org/ Contrast-media.51.0.html\#c269 (consultado feb 2013).

9. Reddan D, LaVille M, GaRovic V. Contrast-induced nephropathy and its prevention: what do we really know from evidence-based findings? J Nephrol 2009; 22: 333-351.

10. KatzBerg R, Lamba R. Contrast-induced nephrotpathy after intravenous administration: fact or fiction? Radiol Clin N Am 2009; 47: 789-800.

11. Cramer BC, Parfrey PS, Hutchinson TA, Baran D, Melanson DM, Ethier RE et al. Renal function following infusion of radiologic contrast material: a prospective controlled study. Arch Intern Med 1985; 145: 87-89.

12. Weisbord S, Mor M, Resnick A, Hartwig K, PaLEVSKY P, FINE M. Incidence and outcomes of contrast-induced AKI following computed tomography. Clin J Am Soc Nephrol 2008; 3: 1274-1281. 